\title{
Distribution and Seasonal Activity of Hard Ticks (Acari: Ixododae) Infesting Domestic Ruminants in Famenin County, Hamadan Province, Iran
}

\author{
Farzaneh Fayazkhoo', Amir Hossein Zahirnia, ${ }^{2, *}$, Zakyeh Telmadarraiy ${ }^{3}$ \\ ${ }^{I}$ MSc in Medical Entomology, Department of Medical Entomology, School of Medicine, Hamadan University of Medical \\ Sciences, Hamadan, Iran \\ ${ }^{2}$ Associate Professor, Department of Medical Entomology, School of Medicine, Hamadan University of Medical Sciences, \\ Hamadan, Iran \\ ${ }^{3}$ Associate Professor, Department of Medical Entomology, School of Medicine, Tehran University of Medical Sciences, \\ Tehran, Iran \\ * Corresponding Author: Amir Hossein Zahirnia, Department of Medical Entomology, School of Medicine, Hamadan \\ University of Medical Sciences, Hamadan, Iran. Email: zahirnia@umsha.ac.ir
}

Received: 01.06.2017

Accepted: 10.09 .2017

How to Cite this Article: Fayazkhoo F, Zahirnia AH, Telmadarraiy Z. Distribution and Seasonal Activity of Hard Ticks (Acari: Ixododae) Infesting Domestic Ruminants in Famenin County, Hamadan Province, Iran. Sci J Hamadan Univ Med Sci. 2017;24(3): 221228. DOI: $10.18869 /$ acadpub. ajcm.24.3.221.

\section{Abstract}

Background and Objective: Hard ticks (Acari: Ixodidae) are one of the most important obligate ectoparasites of vertebrates, belonging to class Arachnida, which transmit a wide range of pathological agents such as viruses, bacteria, and parasites to humans and animals in Iran and around the world. Identifying the distribution of hard ticks in a region is important to monitor their control program, and thereby prevent disease transmission.

Materials and Methods: In this cross-sectional descriptive study, sampling was carried out from different parts of the livestock body during four seasons in four geographical directions and five villages of Famenin County, Hamadan Province, Iran, during 2015-2016. The ticks were initially stimulated by using chloroform solution and then separated from domestic ruminants by forceps. The collected ticks were sent to a laboratory, and then their sex and species were identified by using valid diagnostic keys.

Results: We studied 800 domestic ruminants, including cattle, sheep, and goats, and found $150(18.7 \%)$ cases of infestation. A total of 274 ticks were collected, 259 of which were hard ticks including four genera of Hyalomma, Dermacentor, Repicephlus, and Haemaphysalis. The greatest diversity of species, including Hyalomma scopens (Hy. deteritum), Hyalomma asiaticum, Hyalomma marginatum, and Hyalomma anatolicum belonged to the genus Hyalomma. The frequency rates of Hyalomma, Dermacentor, Rhipicephalus, and Hemaphysalis genera were $73.74 \%, 15.05 \%, 10.03 \%$, and $1.15 \%$, respectively. The highest abundance of ticks was observed in spring (152).

Conclusion: The results of this study indicate the diversity of hard ticks in the region and the highest abundance of ticks in spring. Considering the importance of ticks in disease transmission among humans and domestic ruminants, health authorities and respective organizations should take appropriate health measures to control and combat these external parasites.

Keywords: Domestic Animals; Fauna; Ticks 
of: $10.18869 /$ acadpub.ajcm.24.3.221

\section{بررسى براكندكى و فعاليت فصلى كنههاى سخت (خانواده ايكزوديده) صيدشده از نشخوار كنندكان اهلى در شهر ستان فامنين استان همدان}

\section{فرزانه فياض خو'، امير حسين ظهيرنيا؟،:، زكيه تلمادره ای؟}

' كارشناس ارشد حشره شناسى يزشكى، دانشكده يزشكى، دانشگاه علوم يزشكى همدان، همدان، ايران

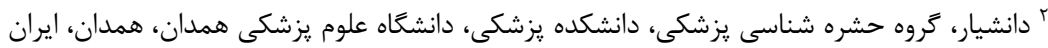

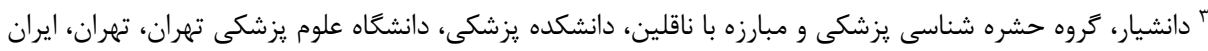
* نويسنده مسئول: اميرحسين ظهيرنيا، گروه حشره شناسى يزشكى، دانشكده يزشكى، دانشگاه علوم يزشكى، همدان، ايران. ايميل:zahirnia@umsha.ac.ir

\begin{tabular}{|c|c|}
\hline 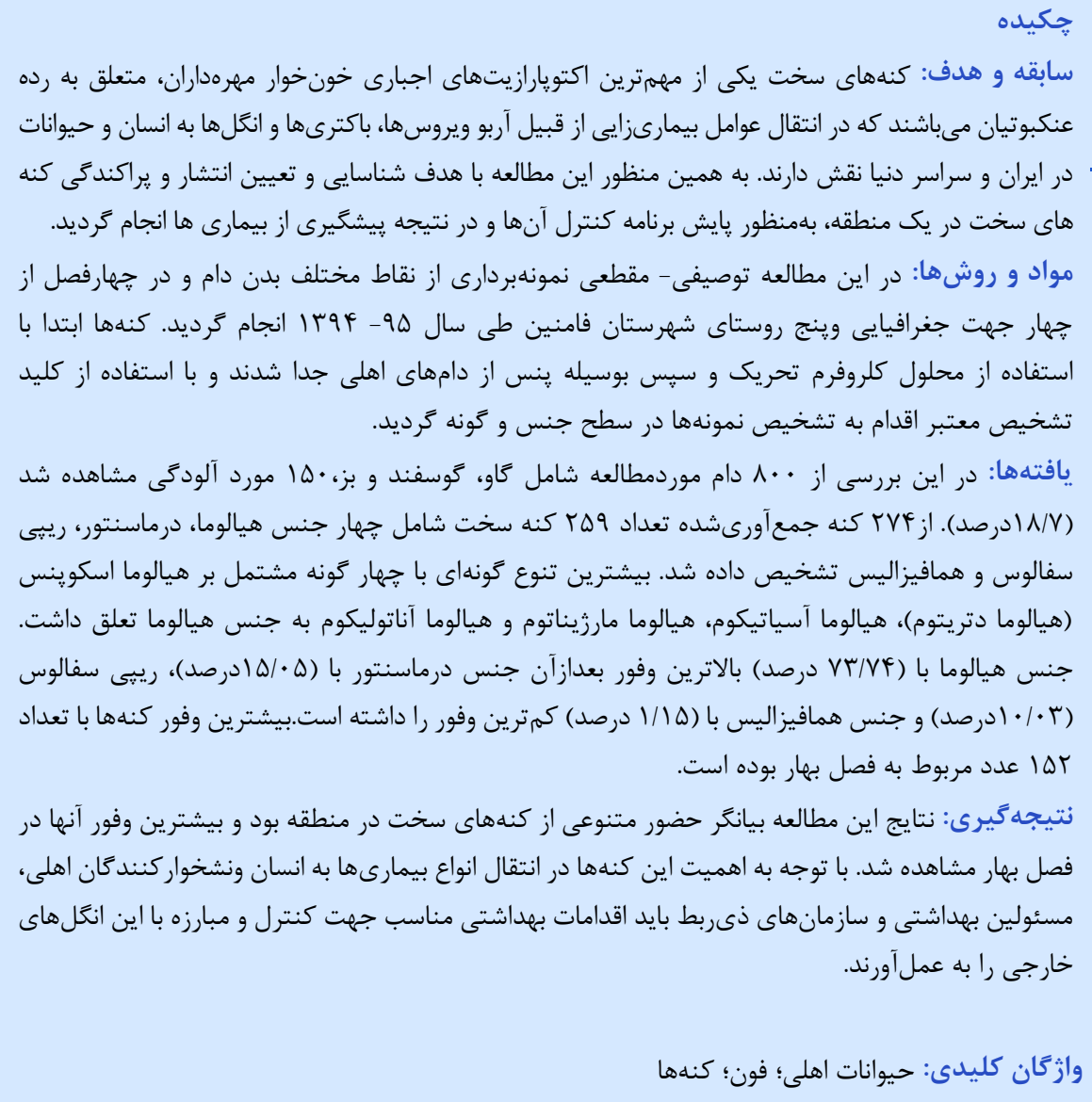 & 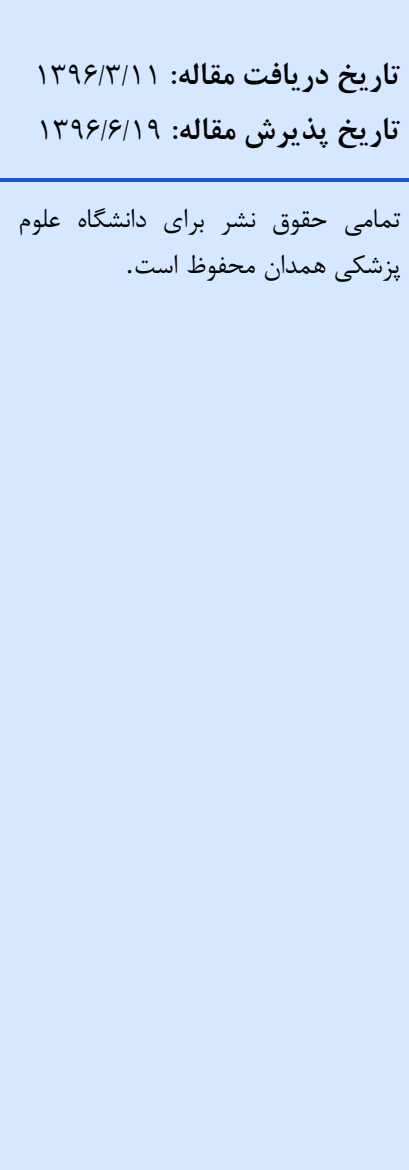 \\
\hline
\end{tabular}

هاى سخت) و آركازيده (كنههاى نرم) قرار دارند كه از هر

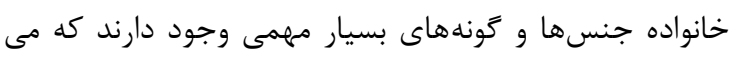

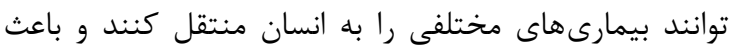

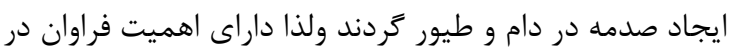

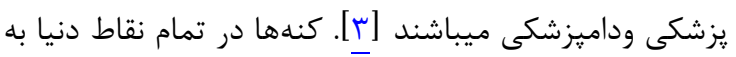

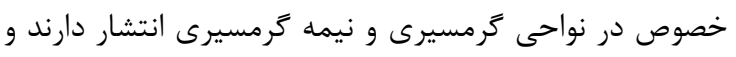

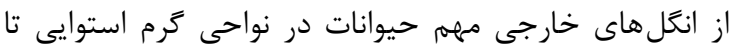

كنههاى ايكزوديده (كنهاى سخت) گَروهى از جانوران

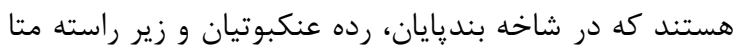

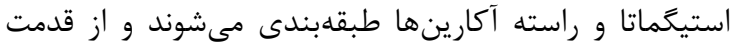

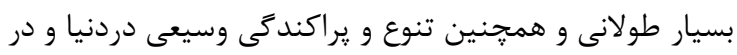

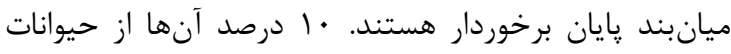

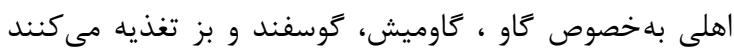

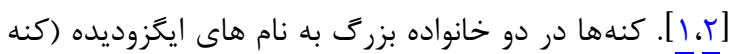


پيشخيرى از بيمارىها داراى اهميت زيادى است. لذا اين

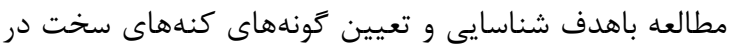

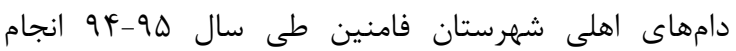
يذيرفت.

\section{مواد و روشها}

اين مطالعه از نوع توصيفى - مقطعى و منطقه مورد مطالعه شهرستان فامنين بود كه در شمال شرق استان همدان قرار

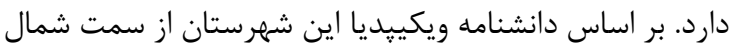
به شهرستان رزن، از سمت جنوب به شهرستان همدان، از سمت دئن

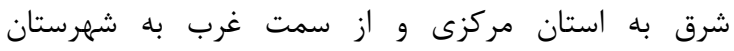

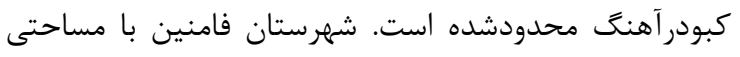
حدود • وسا كيلومترمربع، 9/V درصد از مساحت كل استان

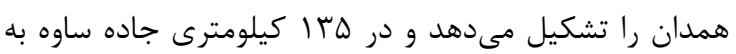

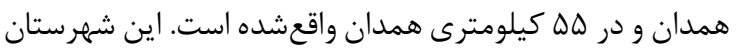
در دشت فامنين- رزن قرار دارد و داراى آبوهواى مدمائ معتدل كوهستانى است. شهر فامنين از شهرهاى قديمى همدان بـ دران

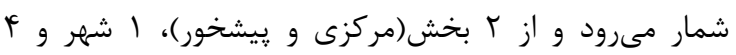

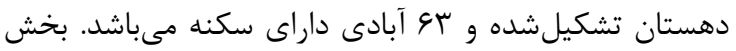

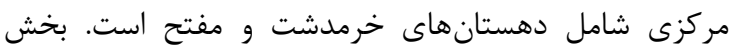

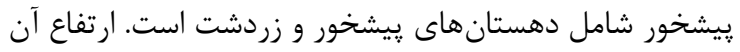

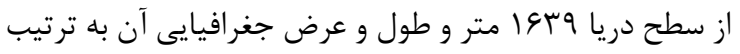

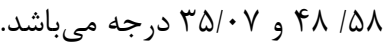

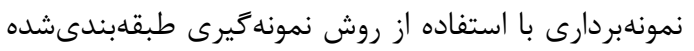
از جهار منطقه (غرب ، شرق ، شمال ، جنوب) شهرستان فامنين

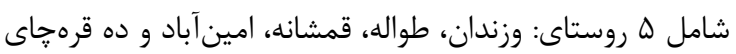

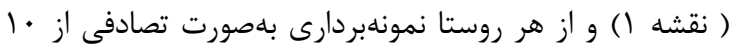

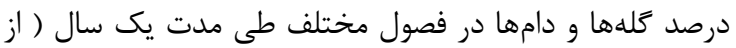

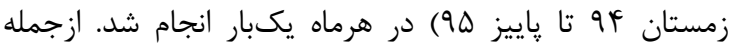
عواملى كه در انتخاب روستاها نقش داشت عادات زيستى مردم، نابيز

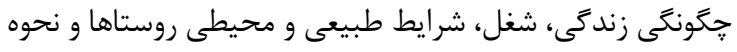

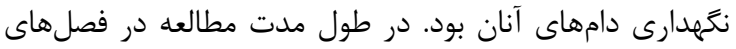

نواحى قطب شمال به شمار مىروند[Y] [ معمولا فصل فعاليت

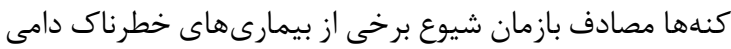

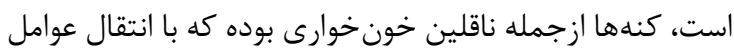
بيمارىزاى مختلف نظير تب خونريزى دهنده كريمه كنگًو تب

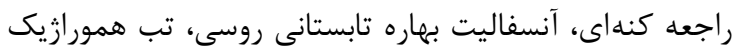

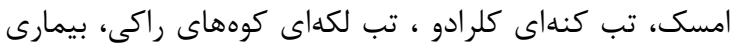

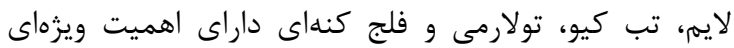
مىباشند [ع، هـ]. در مورد كنههاى ايران مطالعات يراكندهاى انجامشده است

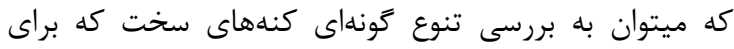

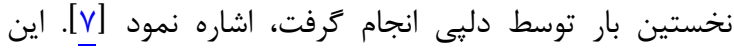

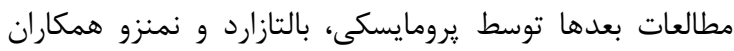

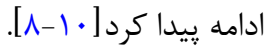

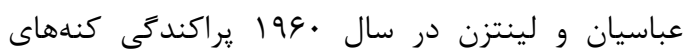
ايكسوديده را در جنوب ايران (سيستان و بلوجستان و جيرفت) دئن

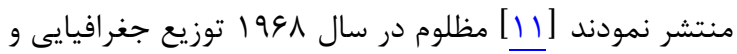

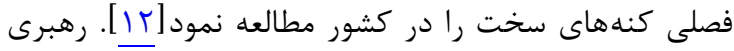
در سال ه9919 جنبههاى اكولوزيكى كنه سنها را در در آذربايجان

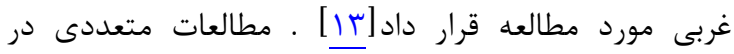

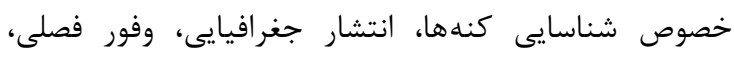

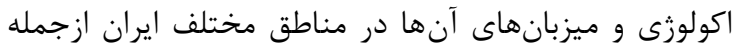

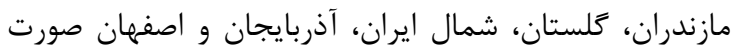

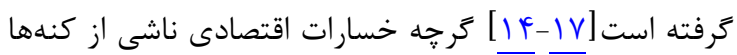
عمدتاً به دليل بيمارىهاى منتقله بلهوسيله آنها است اما زيان

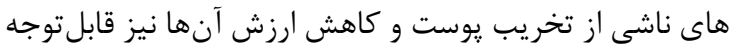

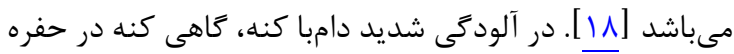

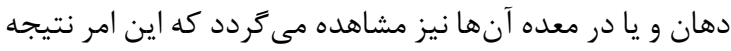

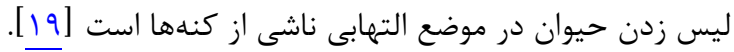

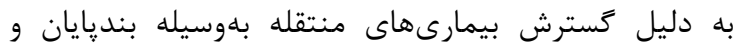

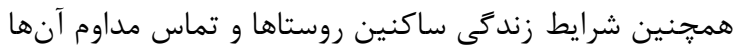

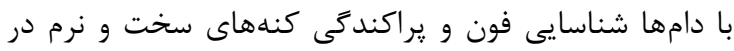

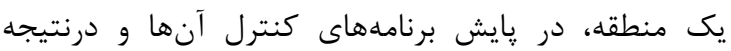

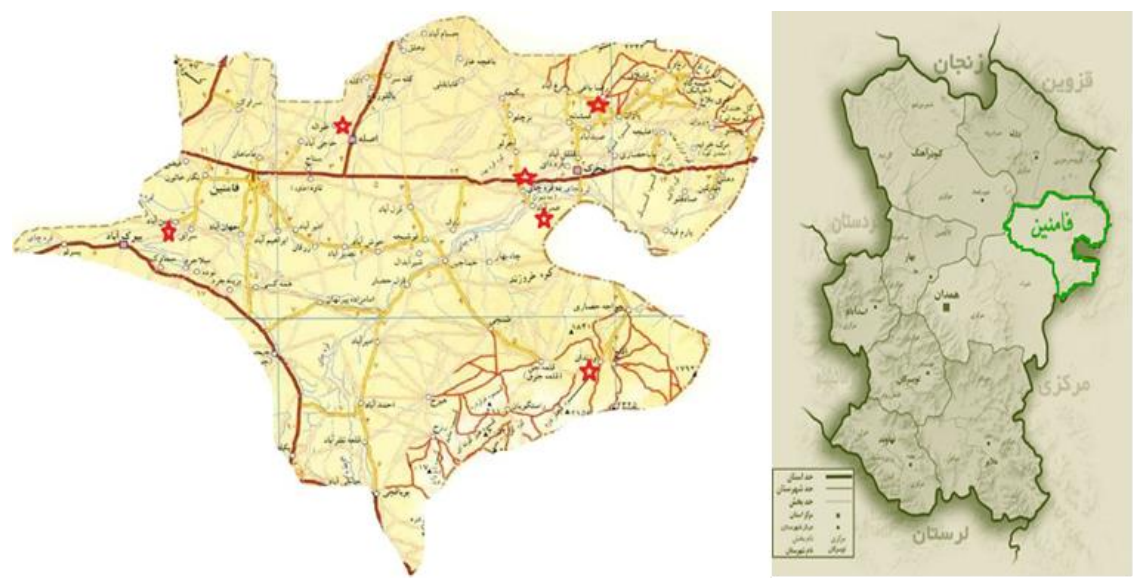

نقشه ا: موقعيت جغرافيايى شهرستان فامنين و روستاهاى تحت مطالعه (كُخ) 


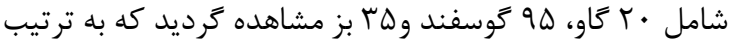

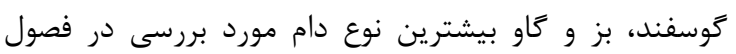

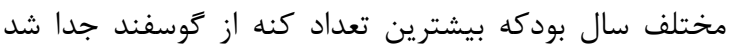
(جدول ( ). از نشخوار كنند

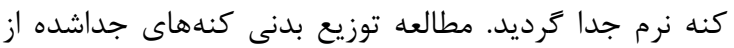
سطح بدن دامهاى منطقه نشان داد كه عمدتاً تجمع كنهها درد

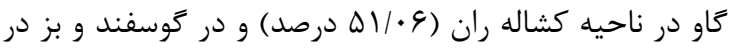

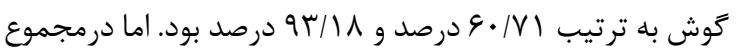

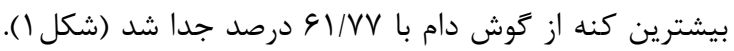

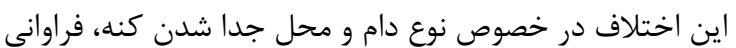

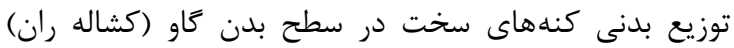

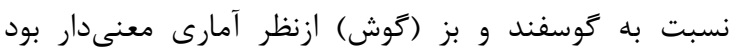
$(\mathrm{P}<\cdot / \cdot \cdot 1)$

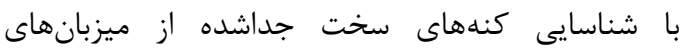

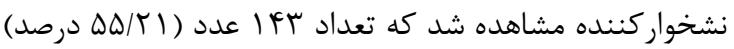

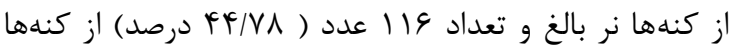

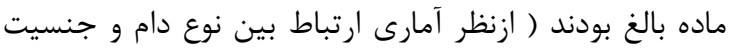

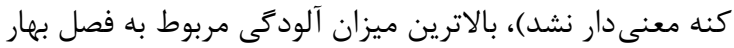

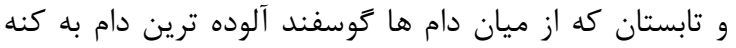

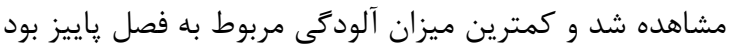

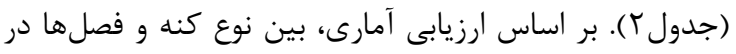

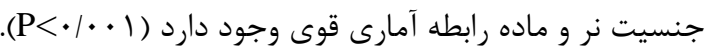

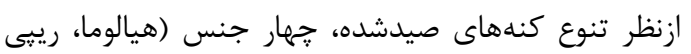

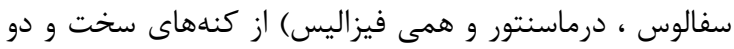

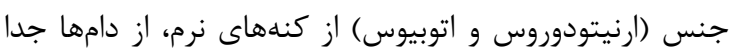

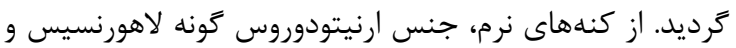
از جنس اتوبيوس گونه مخنينى شناسايى شد.

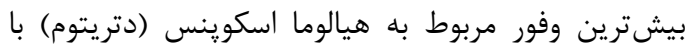

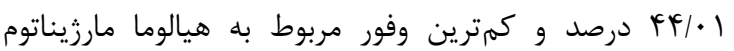

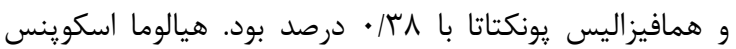

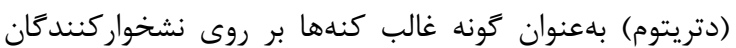
اهلى در فصل بهار در شناخته شد.
بهار و تابستان و ياييز دامها در مرتع بودند و تغذيه آنها نيز از يوشش گیاهى منطقه بود، در فصل زمستان دامها در طويله موردبر سىى قرار گرفتند.

\section{روش جدا كردن و انتقال كنههاى سخت}

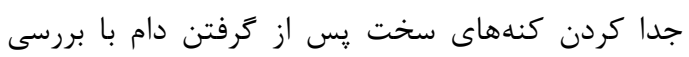

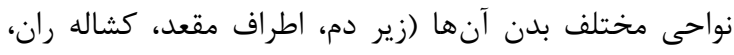

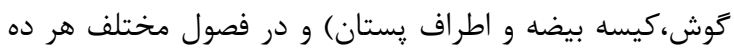
روز يكبار انجام شد. نحوه نمونهبردارى براى خارج كردن كنه لهنه

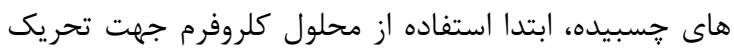
وسيس بيرون كشيدن كنه از بدن دام توسط ينس و وبا زاويه

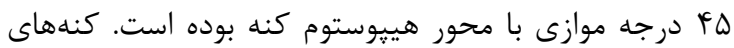

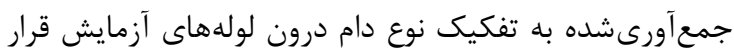
كرفت و مشخصات محل، شامل نام شهرستان، نام روستا و تاريخ

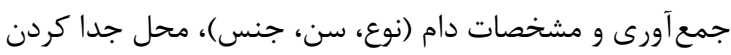

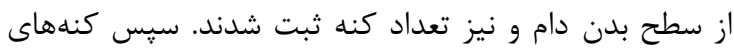

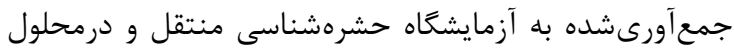

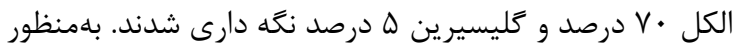
تشخيص، كنهها بهآرامى توسط ينس بردئ برداشتهشده و درو درون

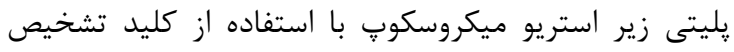

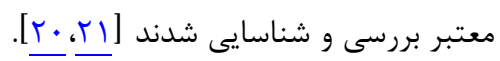

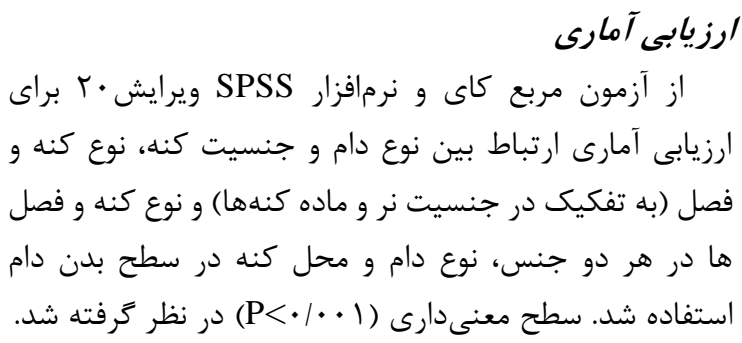

\section{إِفته ها}

از • • دام مورد بررسى در مناطق تحت مطالعه در

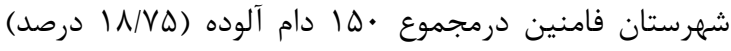

جدول ا: فراوانى و توزيع آلودىى دامها به كنه در شهرستان فامنين

\begin{tabular}{|c|c|c|c|c|c|}
\hline 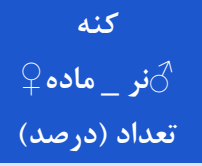 & تعداد (درصد) & 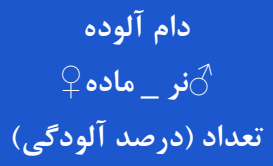 & تعداد (درصد آلودىَ) & تعداد دام & نوع دام \\
\hline $\begin{array}{c}r \cdot-r V \\
I V / T Y-I N / \Lambda \Lambda\end{array}$ & $(|N /| F) F V$ & $(V \cdot-r \cdot) \mid q-q$ & $(T \& \mid \varnothing \varphi) T$. & $V \Delta$ & كاو \\
\hline $\begin{array}{c}V F-q F \\
q r / V q-q Q / V r\end{array}$ & $(\Phi 4 / \Lambda 9) \mid \xi \Lambda$ & $(\Delta \Delta-F \Delta) \Delta r-F r$ & (19/1) $9 \Delta$ & $\Delta q$. & كوسفند \\
\hline $\begin{array}{c}r T-r T \\
|N / q G-| Q / r \Lambda\end{array}$ & $(19 / 91) \mathrm{FF}$ & r & $(T \Delta / q T) T \Delta$ & Iro & بز \\
\hline $\begin{array}{l}119-1 k \mu \\
1 \cdots-1 \ldots\end{array}$ & $(1 \cdots) r \Delta q$ & $(\Delta r-F \wedge) V \Lambda-V r$ & (19) 10. & $\wedge \cdot \cdot$ & جمع كل \\
\hline
\end{tabular}




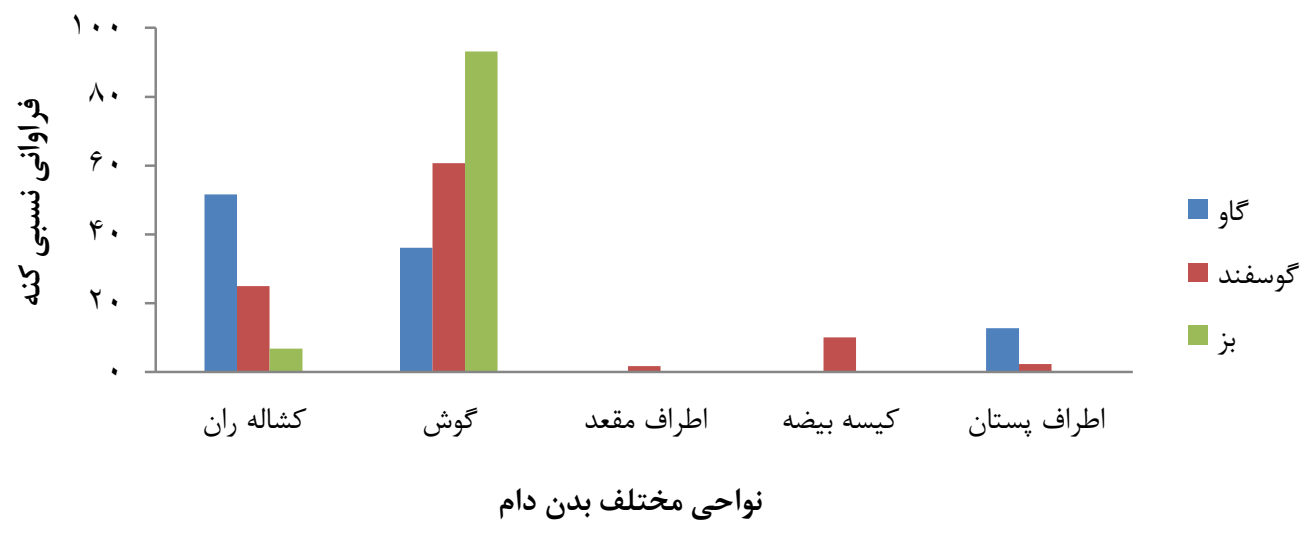

شكل ا: فراوانى نسبى كنهها در نواحى مختلف بدن دام

\begin{tabular}{|c|c|c|c|c|c|c|c|c|c|c|c|}
\hline & جم: & & & & & & تعداد(درصد) & جنس و تونه \\
\hline$q$ & $\hat{0}$ & o & $\hat{\sigma}$ & q & $\hat{\sigma}$ & q & $\hat{0}$ & 운 & $\hat{0}$ & & \\
\hline 01 & Gr & $r$ & Ir & · & $\cdot$ & · & • & $\uparrow \wedge$ & $\Delta \cdot$ & $(f \cdot / \cdot 1) 11 f$ & (Hy.detritum) Hy.scupense \\
\hline f & rr & · & r & · & $\cdot$ & r & $r \Delta$ & 1 & $\Delta$ & ع & Hy.asiaticum \\
\hline · & 1 & $\cdot$ & 1 & · & $\cdot$ & · & · & $\cdot$ & · & $(\cdot / r \wedge) 1$ & Hy.marginatum \\
\hline - & $\Delta$ & $\cdot$ & · & $\cdot$ & $\cdot$ & $\cdot$ & $\Delta$ & $\cdot$ & · & $(1 / 9 \pi) \Delta$ & Hy.anatolicum \\
\hline ra & . & 11 & . & · & $\cdot$ & 1 & . & $r$ & . & (Ir/DI) rQ & Hy.sp \\
\hline 1 & $\Delta$ & 1 & . & · & $\cdot$ & . & . & 9 & $\Delta$ & $(\Delta / \vee 9) \backslash \Delta$ & R.sanguineus \\
\hline f & v & $\cdot$ & · & · & $\cdot$ & · & · & f & v & $(F / T F) \| 1$ & R.bursa \\
\hline 1 & $r q$ & $\cdot$ & . & $\wedge$ & Ir & r & 19 & $\cdot$ & $\cdot$ & $(\mid \Delta / \cdot \Delta) r q$ & D.marginatus \\
\hline . & 1 & $\cdot$ & $\cdot$ & $\cdot$ & $\cdot$ & · & 1 & $\cdot$ & $\cdot$ & $(\cdot / r \Lambda) 1$ & H.punctata \\
\hline r & · & $\cdot$ & · & r & $\cdot$ & $\cdot$ & - & $\cdot$ & $\cdot$ & $(\cdot / V V) Y$ & H.concinna \\
\hline 119 & IFr & 10 & 18 & 1. & ir & 4 & iv & $\Lambda \Delta$ & $9 V$ & $(1 \cdots)$ r $\Delta q$ & مجموع \\
\hline
\end{tabular}

سخت هيالوما، رييى سفالوس، همافيزاليس، درماسنتور و نيز

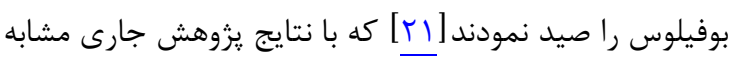

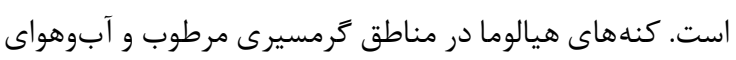

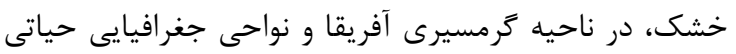

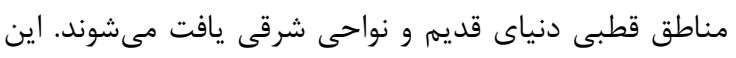

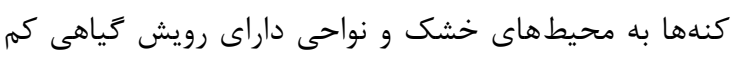

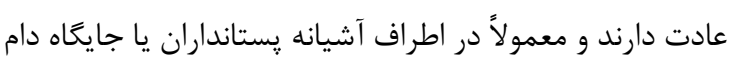

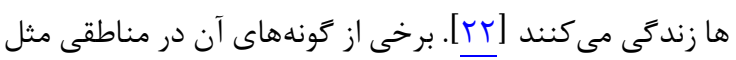

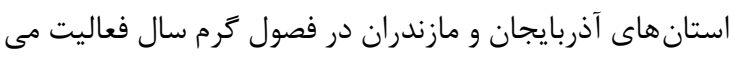

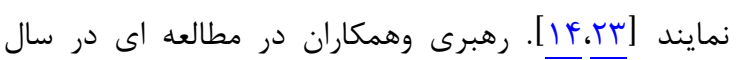

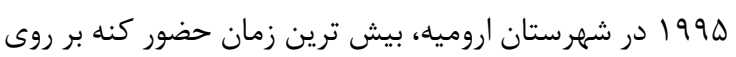

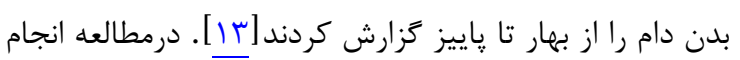

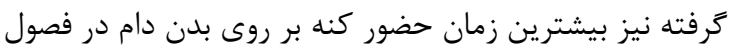

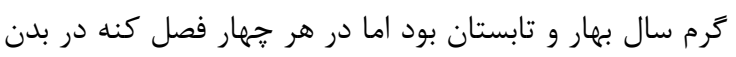
دام ها مشاهده شد. در مطالعه حاضر هيالوما اسكوينس (هيالوما

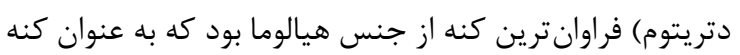

در اين مطالعه با بررسى انتشار كنهها در مناطق مختلف

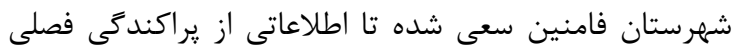
كونهاى مختلف كنه هاى سخت و نوع ميزبان آنها جمع آَرى

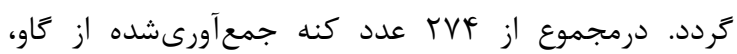

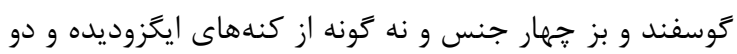

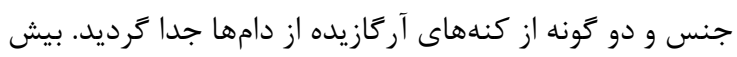
ترين غناى گونهاى با جهار گونه هيالوما اسكوينس إنس (هيالوما دتريتوم)، هيالوما آسياتيكوم، هيالوما مارزيناتوم و هونيان هيالوما

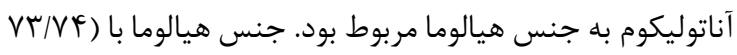

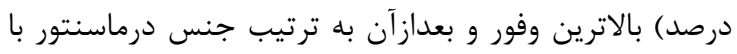

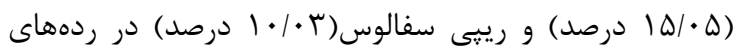

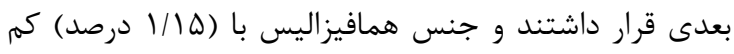

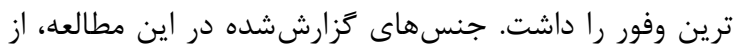

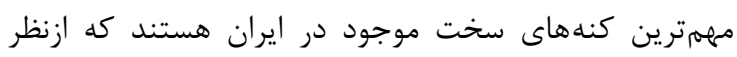

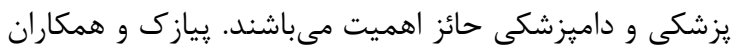

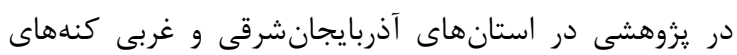




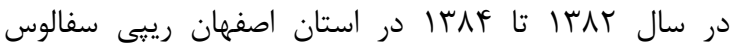
سانگُوئينوس را گونه غالب گوسفندان در مناطق كوهستانى نشان

دادند [IV] رييجى سفالوس بورسا: در مطالعات مختلف انجام ترفته اين

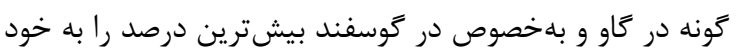

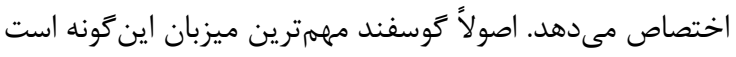

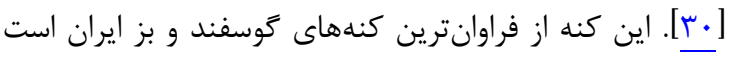

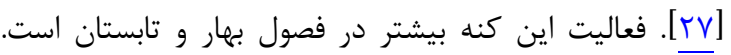

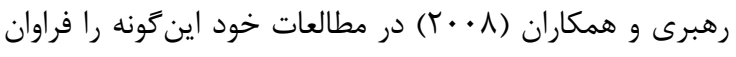

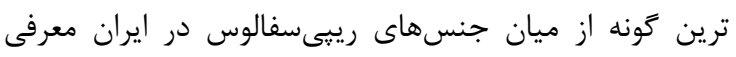

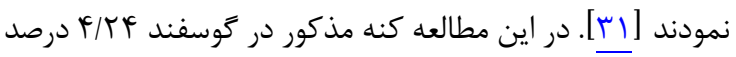
از كل كنههاى جداسازى شده را تشكيل داده است. اين گونه

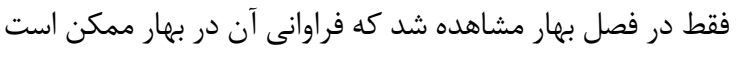

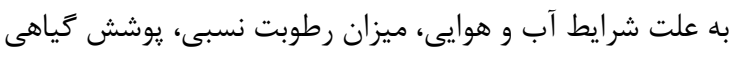

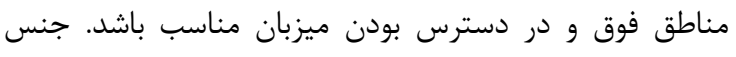

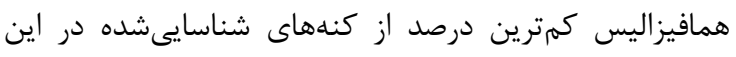

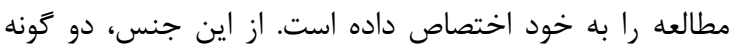

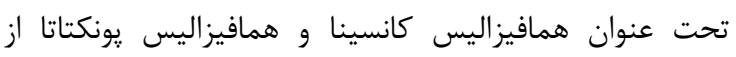
كوسفند جداسازى و شناسايى شد.

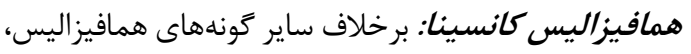

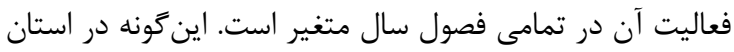

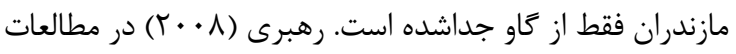

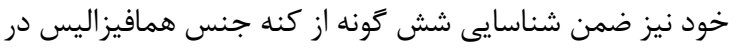

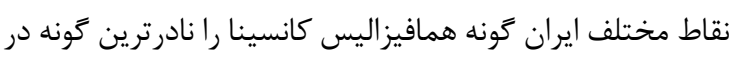

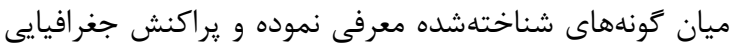

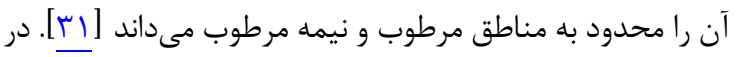

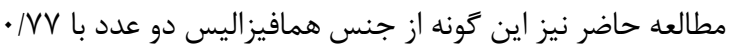

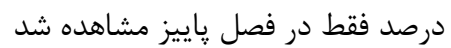

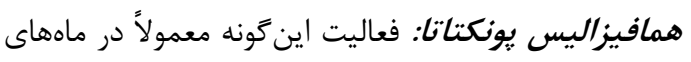

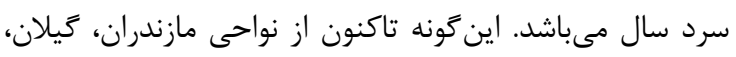
كلستان، خراسان و آذربايجان شناسايىشده است.

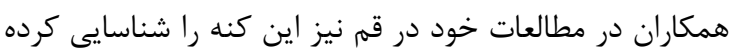

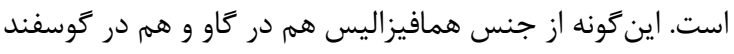

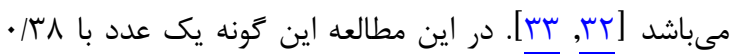
درصد و فقط در تابستان مشاهده شد. در مطالعه انجام

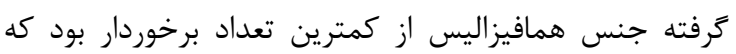

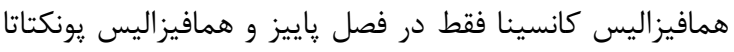
فقط در تابستان مشاهده شد. مطالعه توزيع بدنى كنه هاى جداشده از سطح بدن سند دامهاى نشخواركننده منطقه نشان داد كه بيشترين تجمع كنهاي بهاى

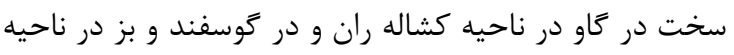

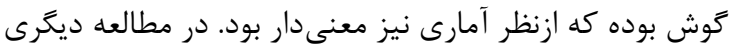

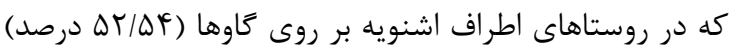

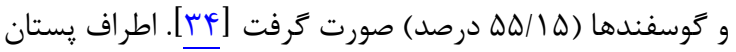

غالب شهرستان فامنين با بالاترين وفور فصلى در بهار مشاهده

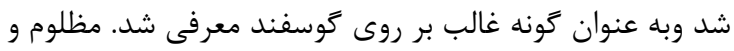

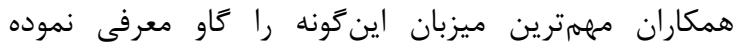

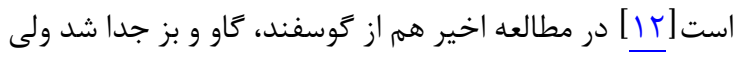

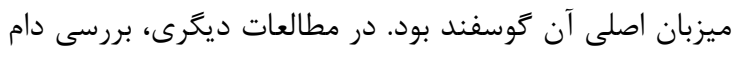

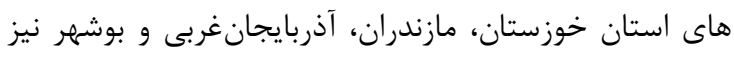

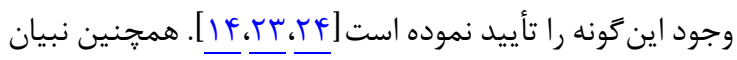

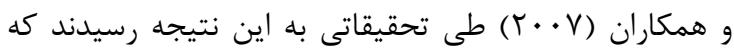

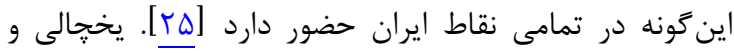

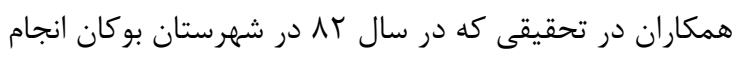

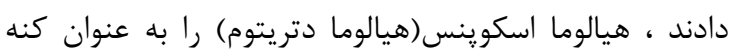

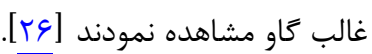

هيالوما مارثيناتوم: اين كونه كمترين درصد از كندهاي

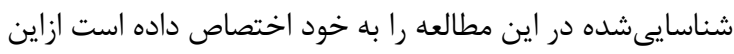

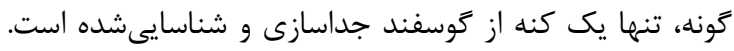
بر اساس تحقيقات مظلوم و همكاران اين گونه فراوانترين كنه از

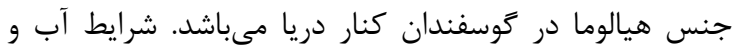

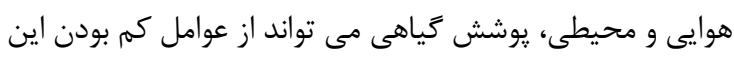

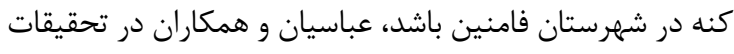
خود مهمترين ميزبان آن را كاو و مظلوم و همكاران مهمرترين بهان

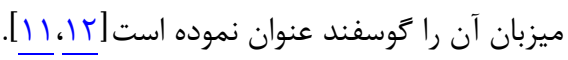

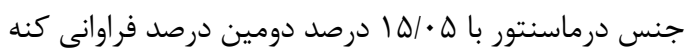

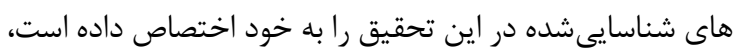

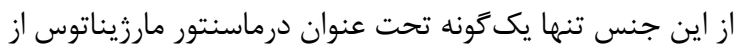

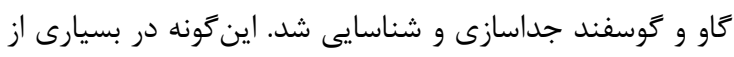

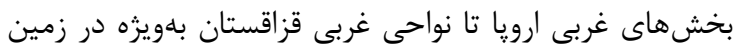

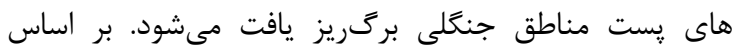

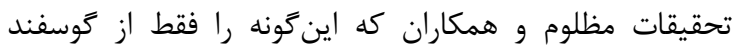
جداسازى نموده اند، مقامى در تحقيقات خود اين گونه را علاوه بر

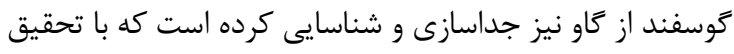

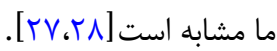

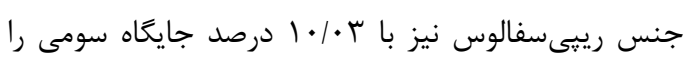

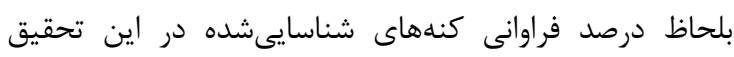

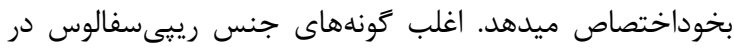

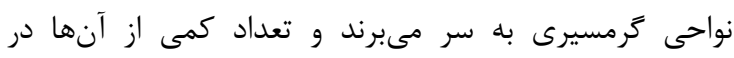
آبوهواى ترمسيرى و ترم مرطوب نواحى شرقى

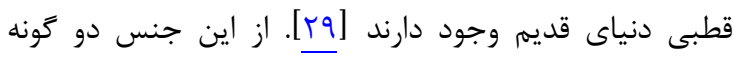
ريبيى سفالوس سانكوينئوس و ريبيى سفالوس بورسا در گَوسفند جداسازى و شناسايى شد.

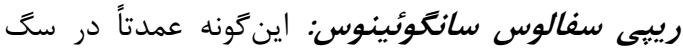

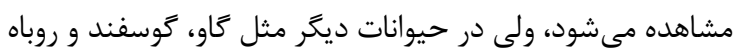

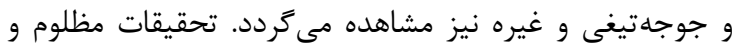

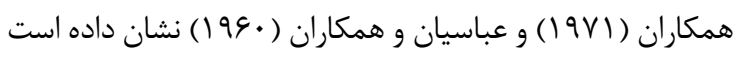

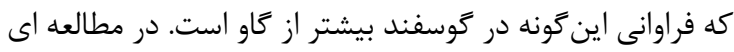




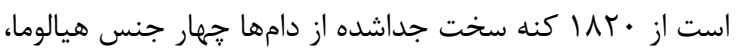

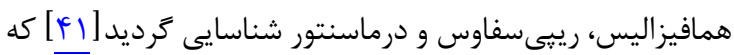

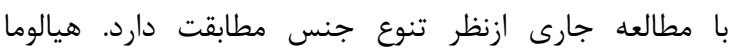

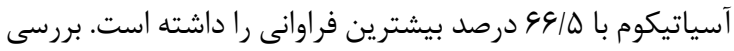

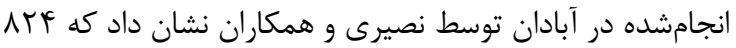

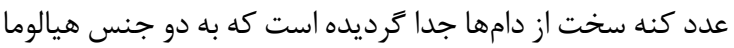

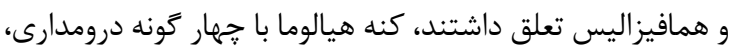

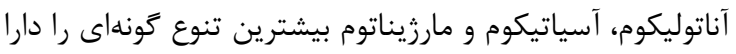

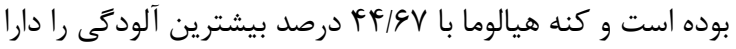

بوده است [FT]

\section{نتيجه تيرى}

مطالعه حاضر و مطالعات مشابه ديكر كه در مناطق مختلف

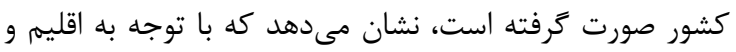
شرايط جغرافيايى هر منطقه مانند شرايط آب و هوايى كوهستانى مرنى

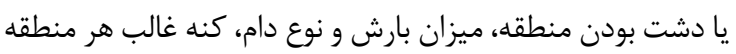

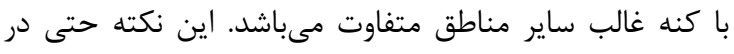

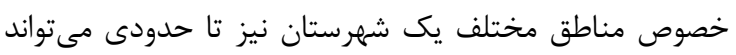

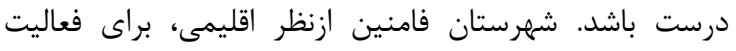

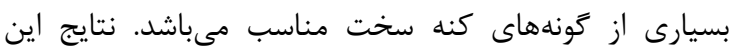
تحقيقات نشان داد كه تنوع گونهاى كنههاى سخت درى درى سطح

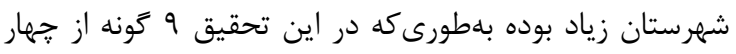

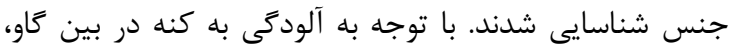

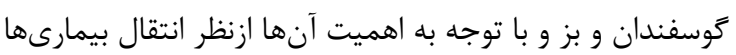

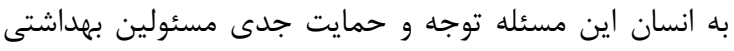

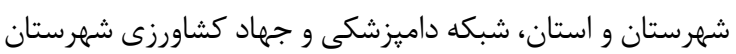

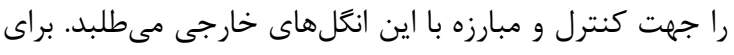

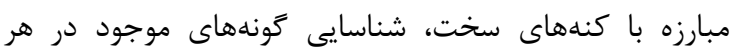

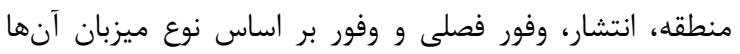

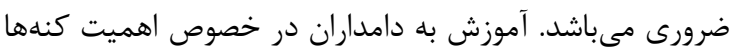

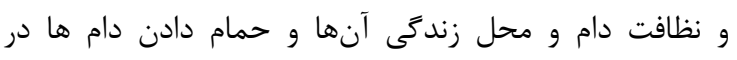
استخرهاى حاوى افت كش مناسب ازجمله راههاى ساده و مؤثر جهت مبارزه و كنترل مى باشد.

\section{تشكر و قلروانى}

اين مقاله بركرفته ار بخشى از يايان نامه كارشناسى ارشد

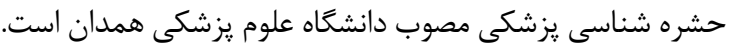

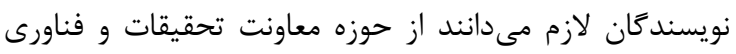

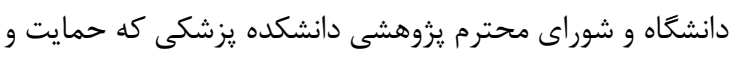

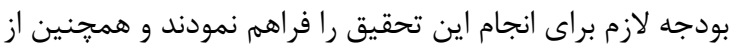

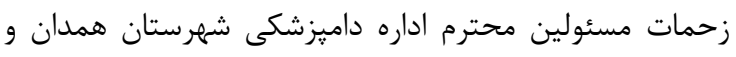
مركز بهداشت شهرستان فامنين تشكر و قدردانى نمايند.ضمنا تضاد منافعى ززارش نشد.
و كشاله ران عمدهترين مواضع تجمع كنههاى سخت گزارش

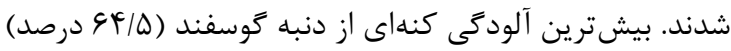

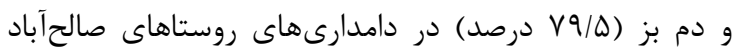

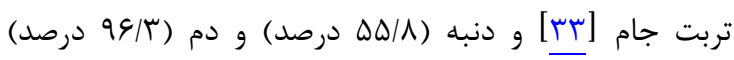

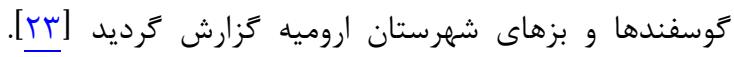

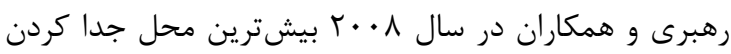

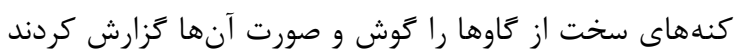

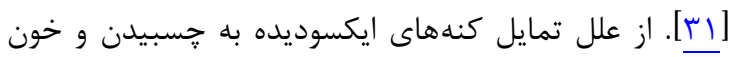

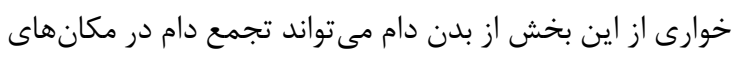

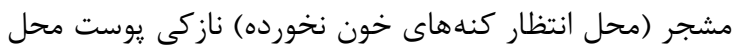

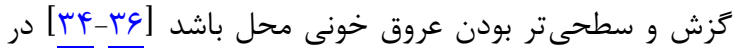

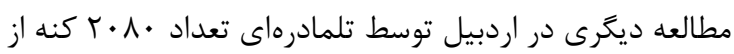

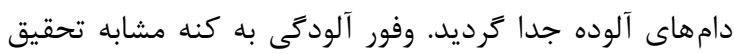

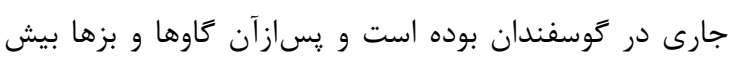

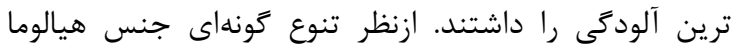

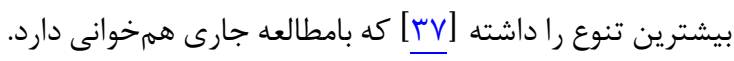
در مطالعه ديخرى توسط بخشايى و همكاران در شهرستان

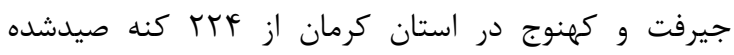

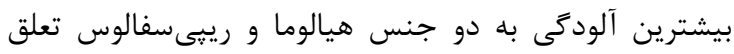

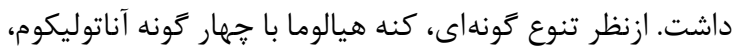

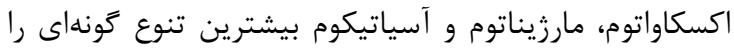

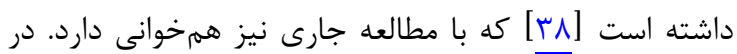
بررسى ديخرى توسط شايقى و همكاران در شهرستانهاى آمل،

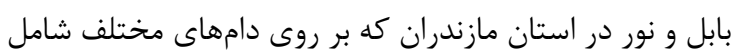

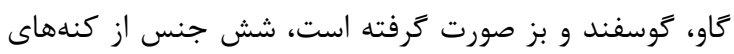

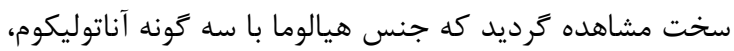

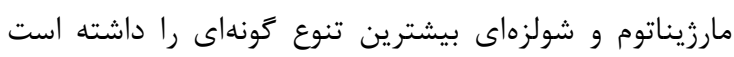

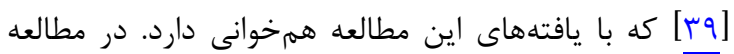

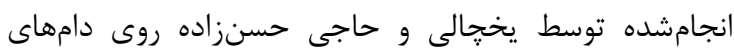

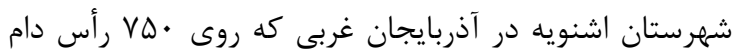

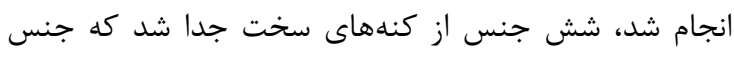

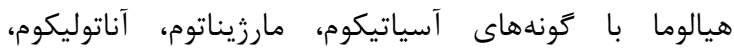

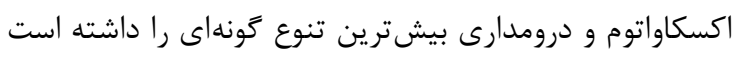

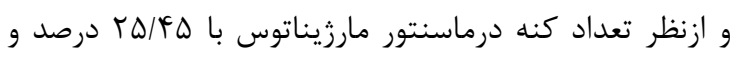

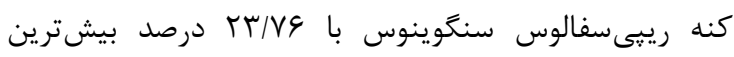

فراوانى را داشته كه با يزوهش جارى مطابقت دارد [بr]س. يزؤهش انجامكرفته توسط شمشاد و همكاران در بوئينزهرا و تاكستان نشان داد كه كنه هيالوما با و آسياتيكوم، اسكورينس (دتريتوم)، درومدارى، مارزيناتوم و شولزه

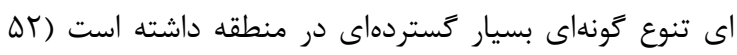

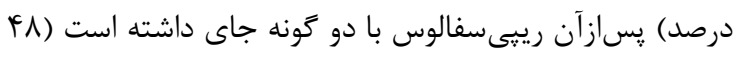

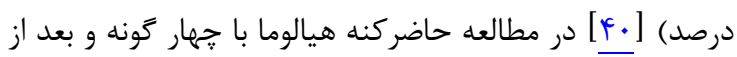

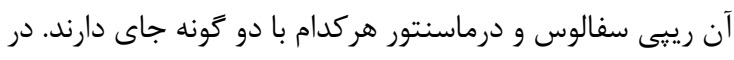

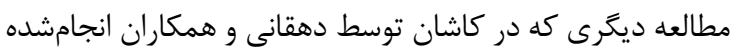




\section{REFERENCES}

1. Wall RL, Shearer D. Veterinary ectoparasites: biology, pathology and control. $2^{\text {nd }}$ ed. Oxford: Oxford University Press; 2001.

2. Ward RD, Service MW. Medical entomology for students. $4^{\text {th }}$ ed. Cambridge: Cambridge University Press; 2008.

3. Taheriyan SM. Vector, biology, morphology, importance and control. Tehran: Sobhan; 2007. P. 102-5. [Persian]

4. Oliver Jr JH. Biology and systematics of ticks (Acari: Ixodidae). Ann Rev Ecol Syst. 1989;20:397-430. DOI: 10.1146/annurev.es.20.110189.002145

5. Parola P, Raoult D. Ticks and tickborne bacterial diseases in humans, an emerging infectious threat. Clin Infect Dis. 2001;32(6):897-928. PMID: 11247714 DOI: $10.1086 / 319347$

6. Raoult D, Roux V. The body louse as a vector of reemerging human diseases. Clin Infect Dis. 1999;29(4):888-911. PMID: 10589908 DOI: $10.1086 / 520454$

7. Delpy L. Revision, par des voies experimentales, du genre Hyalomma C.L.,Koch 1884. Ann Parasitol Hum Comp 1946;21(56):267-93. PMID: 18131390

8. Pervomaisky GS. On the ixodidae of Iran fauna. Trud Voenno Med Akad Krasnoi Armi. 1948;44:35-40.

9. Baltazard M, Bahmanyar M, Safavi G. Sur les differentes de taille observes chez Ornithodorus erraticus. Bull Soc Pathol Exot. 1950;43:444-9.

10. Nemenz H. Ergebnisse der osterreichischen Iran-expedition 1946/1950 Ixodidae. S B Ost Acad Wiss Abt. 1953;162:61-3

11. Abbasian-Lintzen R. A preliminary list of ticks from south eastern of Iran and their distributional data. Acarologia. 1960;2:43-61.

12. Mazlum Z. Hyalomma asiaticum asiaticum Schulze and Schlottke, 1929. Its distribution, hosts, seasonal activity, life cycle, and role in transmission of bovine theileriosis in Iran. Acarologia. 1968;10:437-42.

13. Rahbari S. Studies on some ecological aspects of tick fauna of West Azarbidjan, Iran. J Appl Anim Res. 1995;7(2):18994. DOI: 10.1080/09712119.1995.9706070

14. Razmi GR, Glinsharifodini M, Sarvi S. Prevalence of ixodid ticks on cattle in Mazandaran province Iran. Korean $J$ Parasitol. 2007;45(4):307-10. PMID: 18165714

15. Sofizadeh A, Telmadarraiy Z, Rahnama A, Gorganli-Davaji A, Hosseini-Chegeni A. Hard tick species of livestock and their bioecology in Golestan Province, North of Iran. $J$ Arthropod Borne Dis. 2014;8(1):108-16. PMID: 25629071

16. Telmadarraiy Z, Bahrami A, Vatandoost H. A survey on fauna of ticks in West Azarbaijan Province, Iran. Iran $J$ Public Health. 2004;33(4):65-9.

17. Noaman V, Nabinejad AR, Heidari MR, Khalilifard M. Identification of hard ticks of domestic ruminants in two ecological zones of Isfahan Province. Pajouhesh Sazandegi. 2008;77:88-95. [Persian]

18. Garcia Z. Integrated control of Boophilus microplus in cattle. 11th Internatinal Congress Int. Society for Animal Hygiene, Mexico city, Mexico; 2003.

19. Biswas S. Role of veterinarians in the care and management during harvest of skin in livestock species. Proc National Seminar on Lather Industry in Today's Perspective, Kolkata, India; 2003. P. 62-4

20. Telmadarraiy Z, Vatandoost H, Rafinejad J, Mohebali M, Tavakoli M, Abdigoudarzi M, et al. Distribution of ticks (Ixodidae and Argasidae) family and susceptibility level to cypermethrin in Meshkinshahr district, Ardabil province, Iran. Ardabil Uni Med Sci J. 2009;9(2):127-33.

21. Pyazak N. A preliminary study on the possibility of Lyme disease in the study of ticks I.ricinus. [Master Thesis]. Tehran, Iran: School of Public Health, Tehran University of Medical Sciences; 1991. [Persian]

22. Bakken JS, Dumler JS, Chen SM, Eckman MR, Etta LLV, Walker DH. Human granulocytic ehrlichiosis in the upper
Midwest United States. a new species emerging? JAMA. 1994;272(3):212-8. PMID: 8022040

23. Lak SS, Vatandoost H, Telmadarraiy Z, Mahdi RE, Kia EB. Seasonal activity of ticks and their importance in tick- borne infectious disease in West Azerbaijan, Iran. J Arthropod Borne Dis. 2008;2(2):28-34

24. Zarif-Fard MR, Abdi-Goudarzi M. Identification of ixodidae ticks domestic ruminants in Bousheher, Iran. Arch Inst Razi. 2000;51(1):133-6.

25. Nabian S, Haddadzadeh HR. Current status of tick fauna in north of Iran. Iran J Parasitol. 2007;2(1):12-7.

26. Yakhchali M. Study on Ixodid ticks infestation in cattle, sheep and goats in Bokan suburb, Iran. Iran J Vet Med. 2008;3:100-4. [Persian]

27. Mazlum Z. The ticks of domestic animals in Iran: geographic distribution, host relation and seasonal activity. $J$ Vet Fac. 1971;27(1):1-32

28. Maghami G. External parasites of livestocks in Iran. Arch Insf Razi. 1968;20:81-3.

29. Walker A. Arthropos of humans and domestic animals. New York: Chapman \& Hall; 1994. P. 42-65.

30. Kettle DC. Medical and veterinary entomology. $2^{\text {nd }}$ ed. Cambridge: CAB International; 1995. P. 458-85.

31. Rahbari S, Nabian S, Shayan P, Sedghian M. A study of rhipicephalus species Iran. J Vet Res. 2008;63(4):195-8.

32. Jabari AH, Fesharaki M, Abdi GoudarziM. Identify ticks by ticks isolated from domestic ruminants Qom. Pajuhesh Sazandegi. 2001;14(1):11-3. [Persian]

33. Yakhchali M, Ranjbargarmabolia B. Ixodid ticks fauna in sheep and goats flocks in Torbatejam suburb (South Khorasan province), Iran. Pajuhesh Sazandegi. 2008; 21(3):27-32. [Persian]

34. Yakhchali M, Haji HS. Study on some ecological aspects and prevalence of different species of hard ticks (Acarina: Ixodidae) on cattle, buffalo and sheep in Oshnavieh suburb. Pajuhesh Sazandegi. 2004;17(2):30-5. [Persian]

35. Azizi S, Yakhchali M. Transitory lameness in sheep due to Hyalomma spp. infestation in Urmia Iran. Small Rumin Res. 2006;63(3):262-4. DOI: org/10.1016/j.smallrumres.2005.02.018

36. Muchenje V, Dzama K, Chimonyo M, Raats JG, Strydom PE. Tick susceptibility and its effects on growth performance and carcass characteristics of Nguni, Bonsmara and Angus steers raised on natural pasture. Animal. 2008;2(2):298-304. PMID: 22445024 DOI: $10.1017 /$ S1751731107001036

37. Telmadarraiy Z. Frequency of Ixodidae and Argasidae ticks and determination of their sensitivity in the toxin cypermetryn Meshkinshahr. J Ardabil Univ Med Sci. 2009;9(2):127-33. [Persian]

38. Bakhshai A, Askari N, Etebar F, Ebrahimzade E. Hard ticks fauna in the area of domestic ruminants and Kohnuj Jiroft, Kerman Province, Iran. J Vet Lab Res. 2012;4(1):145-9.

39. Shaeghi M, Yazdi F, Abulhasani M. Distribution of Hard and soft ticks fauna in Mazandaran, Iran. J School Public Health Inst Public Health. 2005;8(3):49-56. [Persian]

40. Shemshad M, Shemshad K, Sadeghi MM, Shokri M, Barmaki A, Baniardalani M, et al. First survey of hard ticks (Acari: Ixodidae) on cattle, sheep and goats in Boeen Zahra and Takistan counties, Iran. Asian Pacific J Tropl Biomed. 2011;2(6):489-92. PMID: 23569956 DOI: 10.1016/S22211691(12)60082-3

41. Dehghani R, Piazak N. Fauna of hard ticks (Acari: Metastigmata) city of Kashan. Const Res. 2003;17(4):19-23.

42. Nasiri A, Telmadarraiy Z, Vatandoost H, Chinikar S, Moradi $\mathrm{M}$, Oshaghi M, et al. Tick infestation rate of sheep and their distribution in Abdanan county, Ilam Province, Iran, 20072008. Iran J Arthropod Borne Dis. 2010;4(2):56-60. PMID: 22808401 